- Case Report

\title{
Gastro Oesophageal Reflux Disease Shadowing Anxiety Disorder Causing Prolonged Exposure to Proton Pump Inhibitor
}

\author{
Nur Sharharni Mohd Tahir', Rosnani Zakaria',*, Nani Draman² \\ ${ }^{1}$ Kepala Batas Health Clinic, Ministry of Health, Seberang Perai, Malaysia \\ ${ }^{2}$ Department of Family Medicine, School of Medical Sciences, Universiti Sains Malaysia, Health Campus, Kubang Kerian, Malaysia
}

Anxiety disorders are the most prevalent psychiatric disorders in the general population. The relationship between dyspepsia and particularly gastroesophageal reflux disease (GERD) and psychiatric comorbidity such as anxiety is poorly defined. However, GERD was noted to be strongly associated and often coincident in onset with generalized anxiety disorder in the community. In this paper, we report the case of an adult man who presented with severe weight loss and underlying GERD, and was later found to have an anxiety disorder as the cause of both.

Keywords: Proton Pump Inhibitor; Gastroesophageal Reflux; Anxiety Disorder; Weight Loss

Received: January 2, 2018, Revised: March 5, 2018, Accepted: April 2, 2018

*Corresponding Author: Rosnani Zakaria https://orcid.org/0000-0002-7093-8278

Tel: +60-9-7676927, Fax: +60-9-7673370, E-mail: rosnaniz74@gmail.com 


\section{INTRODUCTION}

Anxiety disorders are the most prevalent psychiatric disorders in the general population and are the commonest psychiatric disorders associated with dyspepsia. ${ }^{1)}$ Gastroesophageal reflux disease (GERD) can sometimes cause and exacerbate psychiatric disorders; similarly, psychiatric disorders can also precipitate and often coincide in onset with GERD. ${ }^{1)}$ Psychiatric comorbidity is often underrecognized in patients with organic disease, as in the present case. An anxiety disorder is one of the most common causes of recent weight loss. ${ }^{2)}$ Therefore, it is necessary to identify psychiatric comorbidity in GERD patients, as this can help prevent unnecessary use of medications such as a proton-pump inhibitors. ${ }^{3)}$

\section{CASE REPORT}

A 33-year-old man presented with weight loss of $30 \mathrm{~kg}$ in 6 months. His weight at baseline was $109 \mathrm{~kg}$ (body mass index, $39 \mathrm{~kg} / \mathrm{m}^{2}$ ) and decreased to $79 \mathrm{~kg}$. He was diagnosed with GERD 8 months earlier, when esophagogastroduodenoscopy revealed nonerosive esophagitis with a type 1 hiatal hernia. He was under regular follow-up at the surgical outpatient clinic and was taking oral pantoprazole $40 \mathrm{mg}$ daily.

He visited the health clinic several times with complaints of weight loss, bloating, and occasional nonradiating epigastric pain. Vital signs and chest and abdominal examination findings were unremarkable. Results of a complete workup to rule out tuberculosis, thyroid disease, diabetes mellitus, kidney disease, lung disease, urinary infection and anemia were negative. There were no signs or symptoms suggesting lower gastrointestinal tract disease or malignancy. He was only taking pantoprazole and did not smoke or drink to excess. However, since he was diagnosed with GERD and on oral pantoprazole, his weight loss with dyspepsia symptoms were thought to be due to GERD alone. He denied loss of appetite. He was then referred to a family medicine specialist (FMS) due to progressive weight loss with unresolved dyspepsia despite medication. Further evaluation by the FMS revealed that he had been worrying excessively and inappropriately about life events and diseases that might affect his life and cause weight loss. He also reported he was easily fatigued and had myalgias on most days, and was sometimes irritable toward family members and friends over small matters. There were no noted triggering factors. He was still able to go to work except when he had excessive fatigue and tiredness.

He completed a Depression-Anxiety Stress Scale questionnaire for screening and was found to have a moderate anxiety disorder. He was further assessed with the Generalized Anxiety Disorder 7-item (GAD 7) screening tool, and his score of 8 also indicated moderate anxiety. According to the criteria in the diagnostic and statistical manual of mental disorders, fifth edition (DSM V), we diagnosed him as having generalized anxiety disorder due to his symptoms. He was started on oral fluvoxamine $50 \mathrm{mg}$ daily for 1 week and then the dose was increased to $100 \mathrm{mg}$ daily. He was referred to a psychologist for cognitive behavior therapy. On subsequent follow-up, his GERD symptoms had improved, his anxiety was reduced, and he regained about $1 \mathrm{~kg}$ in 2 months. His oral pantoprazole was reduced to as needed use. Fluvoxamine was continued for a total of 9 months and he is still attending regular psychotherapy sessions. He has now regained half of his weight loss. However, because he was previously obese, he was advised to aim for an ideal weight.

\section{DISCUSSION}

Anxiety disorders are the most prevalent psychiatric disorders in the general population. ${ }^{1)}$ One-third of the population is affected by an anxiety disorder during their lifetime. Anxiety disorders are more common in women than in man and the prevalence is highest during midlife. ${ }^{4)}$ Subtypes include GAD, panic disorder with or without agoraphobia, social anxiety disorder, specific phobias, and separation anxiety disorder. Studies in Malaysia showed that the prevalence of GAD was higher than that of other subtypes, at $0.4 \%-5.6 \%$. $^{5}$

GAD is diagnosed based on DSM V criteria, and is characterized by excessive, exaggerated anxiety and worry about everyday life events with no obvious reasons for worry. ${ }^{6)}$ People with symptoms of GAD cannot stop worrying about health, money, family, work, or school. In people with $\mathrm{GAD}$, the worry is often unrealistic or out of proportion to the situation. Daily life becomes a constant state of worry, fear, and dread. Eventually, the anxiety so dominates the person's thinking that it interferes with daily functioning, including work, school, social activities, and relationships.

This disorder seems to play a role in reflux pathogenesis. Both diseases can present in two ways: either one disease precedes the other, or both present concurrently. ${ }^{1)}$ Some studies have systematically investigated the association between GERD and anxiety disorders and noted a high correlation. ${ }^{2,5,7-9)}$ Another study found that psychologically distressed patients are more likely to be found among GERD patients, in whom psychological factors could affect the clinical manifestations of GERD. ${ }^{10)}$

Occasionally, we overlook the diagnosis of a mental disorder in patients with organic diseases. Based on DSM V criteria, symptoms of anxiety disorders must not be attributable to another medical condition. However, in this case, the anxiety disorder caused GERD symptoms and treatment with oral fluvoxamine led to significant improvement in both anxiety and GERD symptoms.

This shows that recognition and management of psychological disorders may aid in the management of unresolved GERD on prolonged treatment with a proton-pump inhibitors. ${ }^{9)}$ Excessive healthcare utilization should alert clinicians to the risk of underlying psychiatric comorbidity.

\section{CONFLICT OF INTEREST}

No potential conflict of interest relevant to this article was reported. 


\section{ORCID}

Nur Sharharni Mohd Tahir: https://orcid.org/0000-0002-3336-4033

Rosnani Zakaria: https://orcid.org/0000-0002-7093-8278

Nani Draman: https://orcid.org/0000-0003-4143-6065

\section{REFERENCES}

1. Mak AD, Wu JC, Chan Y, Chan FK, Sung JJ, Lee S. Dyspepsia is strongly associated with major depression and generalised anxiety disorder: a community study. Aliment Pharmacol Ther 2012;36:800-10.

2. Murtagh J, Rosenblatt J. Murtagh's general practice. 6th ed. North Ryde: McGraw-Hill Australia; 2015.

3. Javadi SA, Shafikhani AA. Anxiety and depression in patients with gastroesophageal reflux disorder. Electron Physician 2017;9:5107-12.

4. Bandelow B, Michaelis S. Epidemiology of anxiety disorders in the 21st century. Dialogues Clin Neurosci 2015;17:327-35.

5. Yang XJ, Jiang HM, Hou XH, Song J. Anxiety and depression in patients with gastroesophageal reflux disease and their effect on quality of life.
World J Gastroenterol 2015;21:4302-9.

6. American Psychiatric Association. The diagnostic and statistical manual of mental disorders: DSM-5. 5th ed. Arlington (VA): American Psychiatric Association; 2013.

7. Jansson C, Nordenstedt H, Wallander MA, Johansson S, Johnsen R, Hveem K, et al. Severe gastro-oesophageal reflux symptoms in relation to anxiety, depression and coping in a population-based study. Aliment Pharmacol Ther 2007;26:683-91.

8. Nunez-Rodriguez MH, Miranda Sivelo A. Psychological factors in gastroesophageal reflux disease measured by scl-90-R questionnaire. Dig Dis Sci 2008;53:3071-5.

9. Sanna L, Stuart AL, Berk M, Pasco JA, Girardi P, Williams LJ. Gastro oesophageal reflux disease (GORD)-related symptoms and its association with mood and anxiety disorders and psychological symptomology: a population-based study in women. BMC Psychiatry 2013;13: 194.

10. Baker LH, Lieberman D, Oehlke M. Psychological distress in patients with gastroesophageal reflux disease. Am J Gastroenterol 1995;90: 1797-803. 